\title{
ESTUDIOS DE VALIDEZ Y CONFIABILIDAD DE LA ESCALA DE COMPRA IMPULSIVA EN JÓVENES Y ADULTOS DE LA CIUDAD DE CÓRDOBA, ARGENTINA
}

\section{VALIDITY AND RELIABILITY STUDIES OF THE BUYING IMPULSIVE SCALE AMONG YOUTH AND ADULTS FROM CORDOBA, ARGENTINA}

\author{
Romina Graciela Revilla \\ Universidad Nacional de Córdoba, Argentina \\ Cristian Acosta \\ Universidad Nacional de Córdoba, Argentina \\ Cecilia Reyna \\ Universidad Nacional de Córdoba, Argentina
}

\begin{abstract}
Resumen: En esta investigación se examinan las propiedades de validez y confiabilidad de la Escala de Compra Impulsiva de Rook y Fisher (1995) en el ámbito local. Además, se aporta evidencia acerca de la relación entre compra impulsiva y arrepentimiento. Participaron estudiantes universitarios (muestra A) y adultos de 18 a 65 años (muestra B) de la ciudad de Córdoba (Argentina). Con la muestra A se realizaron estudios exploratorios y análisis confirmatorios, y se examinó la consistencia interna a través del coeficiente alfa de Cronbach. Con la muestra B, además, se evaluó la correlación entre la compra impulsiva y el arrepentimiento, controlando por la edad de los participantes. Los resultados evidenciaron una estructura unifactorial que explica el $37.17 \%$ de la variabilidad total, en tanto que todos los ítems presentaron cargas factoriales superiores a $.40 \mathrm{y}$ los pesos de regresión estuvieron en el rango $.18-.60$ (muestra A) y $.42-.81$ (muestra B). Se observaron valores alfa de Cronbach aceptables (muestra $\mathrm{A}=.84$ y muestra $\mathrm{B}=.87$ ). $\mathrm{La}$ relación entre compra impulsiva y arrepentimiento mostró ser significativa pero de baja magnitud. En general, los resultados evidencian que la escala presenta adecuadas propiedades psicométricas para ser empleada en el ámbito local.
\end{abstract}

Palabras Clave: compra impulsiva, propiedades psicométricas, arrepentimiento

Abstract: In this research the reliability and validity of the Rook and Fisher's Impulsive Buying Scale (1995) were examined. Evidence about the relationship between impulsive purchase and regret is reported. The samples were composed by university students (sample A) and adults from 18 to 65 years old (sample B) from Córdoba City (Argentina). For sample A, exploratory and confirmatory analyses were performed, and internal consistency was examined by Cronbach's alpha coefficient. For sample B, the correlation between impulsive purchase and regret was also evaluated. The results showed an unifactorial structure that explains the $37.17 \%$ of the total variability, while all items showed factor loadings above .40 and regression weights were in the range .18-.60 (sample A) and .42.81 (sample B). It were observed acceptable Cronbach's alpha coefficient (sample $\mathrm{A}=.84 \mathrm{y}$ sample $\mathrm{B}=.87$ ). The relation between impulsive buying tendencies and regret was significant but low magnitude. Overall, the results show that the scale has adequate psychometric properties to be used locally.

Keywords: impulsive buying, psychometric properties, regret

Este estudio se enmarca en una investigación subsidiada por la Secretaría de Ciencia y Tecnología de la Universidad Nacional de Córdoba. Proyecto de investigación marco: "Toma de decisiones económicas y procesos emocionales: análisis instrumental y experimentos en situaciones de consumo individual e interpersonal".

\section{Introducción}

Desde mediados del siglo pasado comenzó a surgir el interés por el comportamiento de compra impulsiva. Los primeros trabajos se centraron principalmente en la toma de deci- siones en un contexto de compra y la manera en que el ambiente estimulaba las compras no planeadas (Sarabia Sánchez y Schmidt, 2004).

Uno de los primeros autores en abordar la impulsividad en la compra fue Rook (1987), 
quien la definió como un fuerte sentimiento de urgencia irresistible, sin existir una planificación a nivel consciente. Esta impulsividad es difícil de resistir y controlar, ya que permite experimentar un placer anticipado. Posteriormente, Rook y Fisher (1995) plantean que el comportamiento impulsivo puede ser asociado tanto a un comportamiento negativo (primitivo, inmadurez y hasta estupidez), conllevando consecuencias negativas en el campo financiero y también en aspectos personales, asociándose a una bajaautoestima; como así también a situaciones en las que la impulsividad es vista como neutral o de una manera favorable según el punto de vista de la norma social, por ejemplo, decidir comprar impulsivamente algo por una oferta dos por uno o comprar un regalo por impulso debido a que un amigo se encuentra enfermo.

En una era de consumo, resulta de interés estudiar la compra impulsiva, teniendo en cuenta que los estímulos del contexto inmediato pueden ser favorecedores del impulso de compra. Desde sectores de mercado, comprender tal fenómeno favorece la promoción de la compra impulsiva. Con respecto a esto, Rook (1987) señalaba que la mayoría de los compradores tenían notables tendencias impulsivas hacia la compra, influidas por las innovaciones en el mercado, las tarjetas de crédito, las liquidaciones de los negocios, los horarios de apertura de los locales, entre otros.

No obstante, posicionándonos desde un foco distinto al mercado de consumo, avanzar en el conocimiento de la compra impulsiva permitirá no sólo una mejor comprensión del fenómeno y de los factores que lo afectan, sino también promover el uso de herramientas para manejar tal impulso y a su vez, contribuir a formas de consumo más responsables.

Dentro de las definiciones de compra impulsiva, se recuperan inicialmente los aportes de Rook (1987), quien la caracteriza como la experiencia repentina de una urgencia poderosa y persistente para comprar algo inmediatamente. Este impulso a la compra es placenteramente complejo y puede generar un conflicto emocional. Asimismo, Hoch y Loewenstein postulan que la compra impulsiva es el resultado de la tensión entre voluntad y deseo, existiendo tres factores que la desencadenan: "la cercanía física al producto que genera el estímulo, la cercanía temporal y su alta comparación social" (como se citó en Sarabia Sánchez y Schmidt 2004, p. 173).
Rook (1987) identifica una serie de dimensiones que se deben tener en cuenta en la experiencia de impulso a la compra, las cuales se describen a continuación. a) Urgencia espontánea de compra, disparándose por la confrontación visual con el producto o estímulos promocionales (aunque puede ser experimentado sin un estímulo visual). b) Fortaleza del impulso, en tanto que los compradores impulsivos pueden experimentar impulsos psicológicos que estimulan un deseo de actuar inmediatamente. c) Excitación y estimulación, debido a que la compra impulsiva es una fuente de excitación personal y el impulso a la compra es sentido como muy estimulante. d) Sincronicidad, dada por la sensación de los compradores que se encuentran en "el lugar y tiempo correctos". e) Fantasía, ya que muchos sujetos otorgan propiedades cuasi-místicas a los impulsos de compra. f) Hedonismo, debido a que los impulsos de compra pueden estar acompañados por intensos estados de ánimo, positivos o negativos. g) Existencia de conflictos, dado por los interjuegos entre el placer experimentado por la compra y los principios de la realidad, siendo así los impulsos de compra, una fuente de conflictos emocionales y ambivalencia; y finalmente h) Reducida evaluación de las consecuencias, generado por la urgencia del impulso y seguido por la acción inmediata, lo que debilita las consideraciones de las consecuencias de tal comportamiento.

Contrario a esta posición, Pirón (1991) considera que esas dimensiones no son adecuadas, ya que se focalizan más en la compra que en el consumidor, quien se destaca con su comportamiento atípico. Este autor sostiene que la compra impulsiva no se puede definir sin reconocer las reacciones cognitivas y emocionales que produce, a diferencia de Rook (1987), quien considera que son variables de la compra en general.

Siguiendo la misma corriente, Sarabia Sánchez y Schmidt (2004) recuperan los aportes de diversos investigadores (e.g. Pirón, 1991; Rook,1987) para elaborar una nueva propuesta que abarca varias dimensiones de compra impulsiva: a) espontaneidad (ya que no hay una planificación anterior a la compra); b) inductiva (respuesta a estímulos provocadores para la compra, como ofertas); c) emocional (por el placer generado); d) temporalidad (la inmediatez de la compra); e) psicológica (por la misma impulsividad); y f) evaluativa (falta de consideración de las consecuencias). 
En cuanto a la medición de la impulsividad, la literatura reporta la existencia de diversos instrumentos para medirla. Miller (2003) considera que esta diversidad se relaciona con el escaso consenso sobre la definición del constructo, algunas definiciones se centran en la relación entre pensamiento y acciones futuras, mientras que otras comprenden el comportamiento de toma de riesgo. De esta manera, es posible encontrar diferentes instrumentos que pueden medir disímiles facetas de la impulsividad. En ese sentido, Miller (2003) cita algunos ejemplos: la Escala I-7 de Eysenck que evalúa el impulso en sí y el atrevimiento, y la Escala de Impulsividad de Barratt y Patton que comprende componentes motores, cognitivos y de no planificación.

Una de las primeras escalas propuestas para medir el constructo de interés es la Escala de Compra Impulsiva propuesta por Rook y Fisher (1995). Los autores la elaboraron con el fin de indagar la relación entre impulsividad en la compra y los comportamientos de compra de los consumidores. Los participantes eran expuestos a una situación hipotética donde tenían que elegir una de cinco opciones de respuestas caracterizadas por distintos niveles de compra impulsiva. La relación entre el rasgo de compra impulsiva y el comportamiento impulsivo mostró estar moderada por las evaluaciones normativas.

Entre los estudios que han empleado la Escala de Compra Impulsiva de Rook y Fisher (1995), se encuentra el trabajo de Youn y Faber (2000), quienes evaluaron la relación entre la tendencia a la compra impulsiva, rasgos de personalidad (falta de control, absorción y reacción de estrés) y estímulos, en una muestra de estudiantes universitarios norteamericanos. Los resultados indicaron una correlación elevada entre tendencia compra impulsiva y falta de control $(r=.46, p<.001)$, y moderadas puntuaciones con estrés y disposición a abstraerse de la realidad ( $r=.20, p<.05$, en ambos rasgos). En cuanto a los estímulos examinados, siete fueron señalados como potenciales estimuladores de una compra impulsiva: tener dinero, cumpleaños, ofertas, sentirse feliz, navidad, viajar y precios bajos.

Otro de los estudios que han empleado la Escala de Compra Impulsiva de Rook y Fisher (1995), es el conducido por Jones, Reynolds, Weun y Beatty (2003), quienes midieron en una muestra de 261 estudiantes estadounidenses, la tendencia de compra impulsiva general y por otro lado, la tendencia en compra impulsiva específica en relación a la ropa y la música, en una muestra de 261 estudiantes estadounidenses. Los resultados evidenciaron que la tendencia de compra impulsiva general se relaciona de manera significativa y positiva tanto con la tendencia de compra impulsiva para ropa $(r=.72$, $p<.01)$ y para música $(r=.29, p<.01)$.

Uno de los constructos con los cuales se ha relacionado la compra impulsiva es el arrepentimiento. Loomes y Sudgen (1982), sostienen que el arrepentimiento surge cuando las personas comparan los resultados presentes con lo que pudo haber resultado si hubieran elegido otra alternativa, además, se lo puede experimentar cuando la alternativa no seleccionada es la mejor. Asimismo, al igual que Bell (1982), señalan que el arrepentimiento puede afectar a los consumidores en el momento anterior a la decisión de la compra como cuando anticipan tal sensación luego que la decisión ya se encuentra consumada. Por otra parte, Rook (1987) establece que el arrepentimiento experimentado a posterior de una decisión de compra impulsiva puede ser minimizado a partir de la justificación de la decisión de compra.

Inman y McAlister (1994) se basan en la teoría del arrepentimiento para generar predicciones sobre el efecto de un cupón de promoción con fecha de vencimiento en su utilización. Los investigadores hipotetizan y encuentran que si los compradores anticipan la experiencia de arrepentimiento por haber caducado el cupón, se experimenta un mayor arrepentimiento anticipado cuanto más cerca sea la fecha de expiración del cupón, es decir, cuanto más próxima sea la fecha límite del cupón, habrá una mayor probabilidad de utilización por parte de los compradores. Asimismo, cuanto más cercana sea la fecha límite del cupón, se incrementa el potencial de pérdida posible si no se lo utiliza, lo que puede generar motivación a los compradores para comprar y utilizar los cupones.

En un estudio más reciente, Inman y Zeelenberg (2002) avanzan en el análisis del arrepentimiento experimentado tras decisiones repetidas o de cambio. En una serie de estudios con estudiantes universitarios, éstos reportaron que ante experiencias previas positivas los protagonistas (consumidores) de las historias evaluadas que decidían cambiar se arrepentirían más que quienes optaban por repetir su decisión. En cambio, según los estudiantes, ante experiencias previas negativas 
los protagonistas que optaban por cambiar se arrepentirían menos que los que repetían su decisión. De esta manera, los consumidores que cambiaban trataban de evitar pérdidas futuras, mientras que los que repetían su decisión no lo hacían. Simonson (1992), estima que el consumidor puede minimizar el arrepentimiento a partir de la obtención del suficiente conocimiento para poder tomar una decisión informada y tomarse el tiempo necesario para poder examinar las opciones de compra disponibles para poder realizar la transacción de la mejor manera posible. De todas formas, es posible que el consumidor pueda minimizar el arrepentimiento por el placer inmediato de la compra, pero también generar un arrepentimiento a posteriori si este no considera las consecuencias negativas del acto impulsivo (Rook, 1987). Spears (2006) postula que el arrepentimiento luego de una compra impulsiva también puede surgir de la comparación de la realidad (la compra efectivamente realizada) con las alternativas imaginadas.

Tal es la relación entre la compra impulsiva y el arrepentimiento que algunos autores conciben de manera conjunta componentes cognitivos y afectivos de la compra impulsiva y, en ese marco, comprenden al arrepentimiento. En ese sentido, Verplanken y Herabadi (2001) desarrollan una medida general de tendencia de compra impulsiva que en la cual comprenden tanto aspectos cognitivos como la ausencia de planificación, como aspectos afectivos, incluyendo en los mismos al arrepentimiento. Los autores señalan que el arrepentimiento puede ser una respuesta emocional que se puede presentar luego de que se realiza compra no planeada.

Lo expuesto anteriormente denota la necesidad de contar con instrumentos que midan la compra impulsiva y que estén validados localmente. Así, esta investigación se propuso evaluar las características de la Escala de Compra Impulsiva (Rook \& Fisher, 1995) en el contexto local, específicamente, se plantearon los siguientes objetivos: examinar la estructura factorial de la escala y la consistencia interna y, además, aportar evidencia sobre la relación de la compra impulsiva con el arrepentimiento, para lo cual se recurrió a muestras de estudiantes universitarios y población general de la ciudad de Córdoba. A continuación se describen los dos estudios conducidos, primero se describen aspectos metodológicos y luego se presentan los resultados. En la sección final se presenta la discusión general.

\section{Método}

Para esta investigación se recurrió a dos muestras de participantes: estudiantes universitarios y población general de la ciudad de Córdoba, Argentina. Se realilzaron análisis por separado con cada una de las muestras. A continuación se presentan los aspectos metodológicos comunes para ambas muestras y se especifican las singularidades.

\section{Participantes}

Se recurrió a dos muestras: $A)$ estudiantes universitarios y B) población general.

La muestra A estuvo compuesta por 402 estudiantes universitarios con edades comprendidas entre los 18 y 58 años $(M=22.77$, DS $=4.85)$ de ambos sexos [249 (61.9\%) mujeres, $153(38.1 \%)$ varones] seleccionados de manera accidental. Los participantes cursaban distintas carreras de la Universidad Nacional de Córdoba y la Universidad Tecnológica Nacional -regional Córdoba-: abogacía (12.4\%), agronomía $(14.4 \%)$, ciencias económicas $(10.2 \%)$, ingeniería química (17.9\%), psicología $(41.5 \%)$ y ciencias de la educación (3.5\%). En los siguientes años: primero (15.4\%), segundo $(19.4 \%)$, tercero $(32.1 \%)$, cuarto $(10 \%)$, quinto $(19.4 \%)$ y sexto $(3.5 \%)$, sólo un participante no informó el año cursado.

La muestra B estuvo compuesta por 324 personas adultas de 18 a 65 años $(M=32.77$, DS $=10.71)$ de ambos sexos [182 (56.2\%) mujeres y $142(43.8 \%)$ varones], quienes residían en la ciudad de Córdoba (Argentina) y fueron seleccionados de manera accidental. En cuanto al nivel educativo de los entrevistados, la composición fue la siguiente: $3.4 \%$ primario completo, $8.3 \%$ secundario incompleto, $19.4 \%$ secundario completo, $9.9 \%$ terciario incompleto, $12.3 \%$ terciario completo, $30.6 \%$ universitario incompleto, $14.2 \%$ universitario completo, y $1.9 \%$ postgrado. Composición según el nivel socio-económico: $1.9 \%$ marginal, $4.3 \%$ bajo inferior, $21.6 \%$ bajo superior, $25.9 \%$ medio bajo, $23.1 \%$ medio, $13.2 \%$ medio alto y alto (para 18 participantes, $5.6 \%$, no fue posible determinar el NSE). Los participantes de las dos muestras fueron informados oralmente sobre los objetivos del estudio y también vía escrita. En ambas situaciones se destacó el carácter voluntario de su participación, y las condiciones de anonimato y confidencialidad de la información brindada. 


\section{Instrumentos}

Se utilizó la Escala de Compra Impulsiva propuesta por Rook y Fisher (1995). Los autores inicialmente elaboraron 35 ítems sobre compra impulsiva en base a la literatura existente en ese momento y a un estudio fenomenológico previo (Rook, 1987). Esos ítems fueron depurados en un estudio piloto con estudiantes universitarios de ciencias económicas norteamericanos, reduciéndose a 9 ítems. En una nueva muestra de semejantes características, los investigadores evaluaron de manera confirmatoria la estructura de la escala. Aunque la discrepancia entre la matriz teórica y muestral resultó significativa $\left[X^{2}(27, n=212)=49.45, p<.01\right]$, otros indicadores de ajuste resultaron aceptables: $\mathrm{AGFI}=.92$; $\mathrm{CFI}=.97 ; \mathrm{NFI}=.94$. Todos los coeficientes resultaron estadísticamente significativos $(p<.001)$, presentándose en un rango de .60 a .81. En cuanto a la consistencia interna, la misma fue evaluada a través del coeficiente alfa de Cronbach, el cual resultó igual a .88. Para la presente investigación, la escala original fue previamente analizada en un estudio que implicó las siguientes fases: a) Traducción directa de inglés a español por dos especialistas de lengua inglesa y comparación de diferencias, realizando los ajustes necesarios. 2) Estudio piloto con 15 personas adultas para valorar la adecuación cultural, claridad semántica y aspectos gramaticales de los ítems y consigna, tarea que se realizó de manera individual frente a un asistente de investigación entrenado. 3) Discusión al interior del grupo de investigación sobre los resultados del estudio piloto, realizando los ajustes correspondientes, los cuales fueron menores. Los 9 ítems que comprende la escala se puntúan en una escala tipo Likert de 5 puntos ( 1 = muy en desacuerdo, 5 = muy de acuerdo). Con la muestra de estudiantes universitarios se empleó ese formato de puntuación, mientras que con la muestra de población general se empleó un formato de respuesta tipo Likert de 7 puntos $(1=$ muy en desacuerdo, $7=$ muy de acuerdo). Además, se computó el puntaje global bruto sumando la puntuación en cada ítem (previa inversión del ítem 8), con un puntaje mayor siendo indicativo de mayor tendencia de compra impulsiva. A los participantes de esta muestra también se les aplicó la Escala de Arrepentimiento (Reyna, 2013; Schwartz et al., 2002), medida unidimensional que valora la sensibilidad al arrepentimiento en decisiones de la vida real, a través de 5 ítems que se califican sobre una escala tipo Likert de 7 puntos ( 1 = totalmente en desacuerdo, $7=$ totalmente de acuerdo). La suma de todos los ítems arroja una puntuación total donde un puntaje mayor indica mayor tendencia al arrepentimiento. En este estudio, el índice alfa de Cronbach para esta escala fue de .72. Finalmente, en la muestra A se recuperó información sobre datos sociodemográficos a través de un cuestionario elaborado ad-hoc. Mientras que en la muestra B se empleó un cuestionario sobre datos socio-demográficos, a partir del cual se estimó el NSE en base a la relación de aportantes y miembros del hogar, nivel educativo, ocupación y cobertura de salud del principal sostén del hogar, e indicadores de indigencia (Comisión de Enlace Institucional, AAM-SAIMO-CEIM, 2006).

\section{Procedimiento y análisis de datos}

Con la muestra A se procedió de la siguiente manera. Primero, se solicitó autorización a los docentes a cargo de distintas asignaturas. Tras ello, se invitó a los estudiantes a participar en el estudio. Los participantes completaron los cuestionarios individualmente en sus correspondientes grupos de clase, en horario y espacio áulico. Con la muestra $\mathrm{B}$, los participantes fueron invitados a participar a través de convocatorias en espacios públicos. Luego de la explicación de los objetivos del estudio y las condiciones de participación, se solicitó información sobre datos socio-demográficos. Tras ello, se explicó la forma de completar los restantes cuestionarios y se brindaron ejemplos, asegurándose que el participante había comprendido totalmente la tarea. Los participantes completaron individualmente los cuestionarios, los cuales fueron retirados posteriormente en horario y espacio definido por el participante.

En ambos casos la administración fue realizada por investigadores formados y asistentes de investigación previamente entrenados. Las escalas formaban parte de un conjunto de instrumentos que no son objeto de este estudio.

Los datos fueron analizados con el programa estadístico SPSS 20 y AMOS 19. Primero, se realizó una exploración inicial de los datos con el fin de identificar valores perdidos y extremos y examinar la forma de distribución de los ítems de la Escala de Compra Impulsiva. Segundo, se evaluó la estructura de la escala de manera exploratoria y confirmatoria en el 
caso de la muestra A, y sólo confirmatoria en el caso de la muestra B. Tercero, se estimó la consistencia interna a través del coeficiente alfa de Cronbach. Cuarto, sólo con la muestra B, se evaluó la correlación entre la compra impulsiva y el arrepentimiento, controlando por la edad de los participantes.

\section{Resultados}

A continuación se exponen los resultados obtenidos agrupados según las muestras empleadas.

\section{Muestra A: estudiantes universitarios}

a) Análisis preliminar

Un análisis inicial evidenció que seis participantes no habían respondido a dos ítems, y que otros 22 participantes no respondieron a 1 ítem. Además, se observó que ninguna variable presentaba más del $5 \%$ de datos ausentes. Según el test MCAR de Little (Missing Completely at Random) la distribución de los mismos fue completamente aleatoria $\left(X^{2}(85)=96.097\right.$, $p=.193$ ), por lo que se optó por imputarlos por la media.

No se apreciaron casos atípicos univariados $(Z> \pm 3.29)$ y tampoco multivariados (Tabachnik y Fidell, 2001). El análisis de distribución de las variables, como se aprecia en la Tabla 1 , muestra que los ítems presentan excelentes $( \pm 1)$ valores de asimetría y curtosis (George \& Mallery, 2001), a excepción del ítem "'Compro primero y pienso después' me describe bien" que presentó valores aceptables $( \pm 1.5)$ de asimetría y curtosis, y el ítem "Compro cosas según cómo me siento en el momento" que mostró un valor aceptable de curtosis. También se comprobó la ausencia de multicolinealidad a través de la inspección de las correlaciones entre ítems, las cuales resultaron menores a .90 (Tabachnick \& Fidell, 2001).

\section{b) Estructura factorial}

La muestra fue dividida aleatoriamente en dos mitades. La muestra $1(n=201)$ fue empleada para el análisis factorial exploratorio (AFE) y la muestra $2(n=201)$ para el análisis factorial confirmatorio (AFC).

Para el Análisis Factorial Exploratorio (AFE) se utilizó el método de Ejes Principales. El índice de adecuación muestral KMO fue .832, el test de esfericidad de Bartlett fue significativo $\left[\mathrm{X}^{2}\right.$ aprox. $(36, n=201)=618.602, p<.000]$, lo cual garantizó la factibilidad del análisis factorial. La regla de Kaiser-Gutman sugirió la extracción de dos factores. No obstante, debido a que con dicha regla se tiende a sobreestimar las dimensiones subyacentes (Cohen y Swerdlik, 2006), y que la estructura bidimensional resultaba compleja (p.e., ítems con semejante carga factorial en los dos factores), se recurrió a la interpretación del gráfico de sedimentación, el cual sugirió la existencia de un factor. Se reiteró el análisis y se solicitó la extracción de un factor, el cual explica el $37.17 \%$ de la varianza total. Todos los ítems mostraron cargas factoriales mayores a .40 (Tabla 2).

Para el Análisis Factorial Confirmatorio (AFC) se empleó el método de estimación de máxima verosimilitud. El análisis inicial indicó que el estadístico de discrepancia era significativo $\left[\mathrm{X}^{2}(27, n=201)=83.533, p<.000\right]$. A partir de los índices de modificación y los valores esperados de cambio del parámetro provistos por el software utilizado, se permitieron tres correlaciones entre errores de las variables,

Tabla 1

Muestra A: estudiantes universitarios - Estadísticos descriptivos de los items de la Escala de Compra Impulsiva

\begin{tabular}{lcccc}
\hline & $M$ & $D S$ & Asimetría & Curtosis \\
\hline 1. A menudo compro cosas de manera espontánea & 3.16 & 1.12 & -0.22 & -0.75 \\
2. "Ya mismo" describe la forma en que compro las cosas & 2.43 & 1.08 & 0.42 & -0.38 \\
3. A menudo compro cosas sin pensar & 2.40 & 1.18 & 0.59 & -0.59 \\
4. Si veo algo que quiero, lo compro & 2.84 & 1.11 & 0.00 & -0.75 \\
5. "Compro primero y pienso después" me describe bien & 1.92 & 1.06 & 10.23 & 1.06 \\
6. Algunas veces soy un poco insensato (irracional, ilógico) comprando & 2.16 & 1.18 & 0.87 & -0.22 \\
7. Compro cosas según cómo me siento en el momento & 2.65 & 1.26 & 0.20 & -1.12 \\
8. Planifico cuidadosamente la mayoría de las compras & 2.65 & 1.11 & 0.43 & -0.47 \\
9. Algunas veces soy un poco imprudente con lo que compro & 2.62 & 1.16 & 0.30 & -0.84 \\
\hline
\end{tabular}


teniendo en cuenta que fueron medidas con un mismo instrumento. Si bien la discrepancia disminuyó, continuó siendo significativa $\left[X^{2}(21, n=201)=44.689, p<.006\right]$. No obstante, según otros índices, el ajuste es adecuado: $\mathrm{X}^{2} / \mathrm{gl}=1.862 ; \mathrm{AGFI}=.916 ; \mathrm{NFI}=.935 ;$ $\mathrm{TLI}=.953 ; \mathrm{CFI}=.968 ; \mathrm{RMSEA}=.066$. Finalmente, se evaluaron los coeficientes de regresión estandarizados, los cuales estuvieron en el rango de .18 a .60 y resultaron todos significativos $(p<.001$, Tabla 2).

\section{c) Consistencia interna}

Finalmente, con toda la muestra de estudiantes universitarios $(n=402)$, se estimó la consistencia interna a través del coeficiente alfa de Cronbach, el cual resultó igual a .846, lo cual es considerado aceptable (Tabla 2).

\section{Muestra B: población general}

\section{a) Análisis preliminar}

El análisis inicial evidenció que ocho participantes no habían respondido los ítems de la escala bajo estudio, por lo cual se optó por excluir esos participantes de los análisis posteriores. En cuanto al resto de los participantes, 14 no respondieron a un ítem, y siete participantes no respondieron a dos ítems. Además, se observó que ninguna variable presentaba más del $5 \%$ de datos ausentes. Según el test MCAR de Little la distribución de los mismos fue completamente aleatoria $\left[X^{2}(98)=91.533, p=.664\right]$, por lo cual se optó por reemplazar los valores ausentes con la media.

No se apreciaron casos atípicos univariados $(Z> \pm 3.29)$, mientras que un caso mostró atipicidad múltiple $(p<.001)$ (Tabachnik \& Fidell, 2001) y se optó por excluirlo de la muestra, resultando 315 casos para los análisis que se desarrollaron a continuación. Luego, se analizó la distribución de las variables. Se observaron valores excelentes de asimetría y curtosis $( \pm 1$, George \& Mallery, 2001), los cuales se pueden observar en la Tabla 3. También se comprobó la ausencia de multicolinealidad a través de la inspección de las correlaciones entre ítems, las cuales resultaron menores a .90 (Tabachnick \& Fidell, 2001).

\section{b) Estructura factorial}

La estructura factorial fue evaluada de manera confirmatoria. Se utilizó el método de estimación de máxima verosi-

Tabla 2

Muestra A: estudiantes universitarios - Saturación factorial de los items de la Escala de Compra Impulsiva según distintos análisis factoriales y alfa de Cronbach si el ítem es eliminado

\begin{tabular}{lccc}
\hline & AFE & AFC & $\begin{array}{l}\text { alfa de Cronbach si } \\
\text { el ítem es eliminado }\end{array}$ \\
\hline 1. A menudo compro cosas de manera espontánea & .48 & .61 & .84 \\
2. "Ya mismo" describe la forma en que compro las cosas & .56 & .72 & .83 \\
3. A menudo compro cosas sin pensar & .83 & .78 & .81 \\
4. Si veo algo que quiero, lo compro & .54 & .62 & .83 \\
5. "Compro primero y pienso después" me describe bien & .71 & .74 & .82 \\
6. Algunas veces soy un poco insensato (irracional, ilógico) comprando & .77 & .70 & .82 \\
7. Compro cosas según cómo me siento en el momento & .48 & .62 & .84 \\
8. Planifico cuidadosamente la mayoría de las compras & .45 & .42 & .85 \\
9. Algunas veces soy un poco imprudente con lo que compro & .55 & .55 & .84 \\
\hline
\end{tabular}

Nota. AFE = análisis factorial exploratorio con método de extracción de ejes principales $(n=201)$; AFC = análisis factorial confirmatorio, coeficientes de regresión estandarizados $(n=201)$. El análisis de consistencia interna se realizó en base a la muestra total $(n=402)$.

Muestra B: población general - Estadísticos descriptivos de los items de la Escala de Compra Impulsiva

\begin{tabular}{|c|c|c|c|c|}
\hline & M & $D S$ & Asimetria & Curtosis \\
\hline 1. A menudo compro cosas de manera espontánea. & 4.32 & 1.71 & -0.32 & -0.77 \\
\hline 2. "Ya mismo" describe la forma en que compro las cosas. & 3.34 & 1.70 & 0.28 & -0.90 \\
\hline 3. A menudo compro cosas sin pensar. & 3.22 & 1.72 & 0.38 & -0.84 \\
\hline 4. Si veo algo que quiero, lo compro. & 4.11 & 1.78 & -0.16 & -0.95 \\
\hline 5. "Compro primero y pienso después" me describe bien. & 2.68 & 1.71 & 0.88 & -0.16 \\
\hline 6. Algunas veces soy un poco insensato (irracional, ilógico) comprando. & 3.04 & 1.80 & 0.62 & -0.64 \\
\hline 7. Compro cosas según cómo me siento en el momento. & 3.19 & 1.83 & 0.37 & -1.01 \\
\hline 8. Planifico cuidadosamente la mayoría de las compras. & 3.70 & 1.81 & 0.26 & -0.92 \\
\hline 9. Algunas veces soy un poco imprudente con lo que compro. & 3.32 & 1.73 & 0.26 & -0.86 \\
\hline
\end{tabular}


militud. El análisis inicial indicó que el estadístico de discrepancia era significativo $\left[X^{2}(27, n=315)=111.598, p<.000\right]$, ytambiénotrosíndices indicaban un mal ajuste de los datos al modelo $\left[\mathrm{X}^{2} / \mathrm{gl}=4.133 ; \mathrm{AGFI}=.867 ; \mathrm{NFI}=.903 ;\right.$ $\mathrm{TLI}=$.899; RMSEA =.1]. Debido a ello, se procedió a examinar los índices de modificación y los valores esperados de cambio del parámetro, tras lo cual se permitieron cuatro correlaciones entre los errores de las variables. Si bien la discrepancia disminuyó, continuó siendo significativa $\left[X^{2}(23, n=315)=54.372, p<.000\right]$, el ajuste según otros índices fue aceptable: $X^{2} / g l=2.364$; $\mathrm{AGFI}=.925 ; \mathrm{NFI}=.953 ; \mathrm{TLI}=.956 ; \mathrm{CFI}=.972$; RMSEA $=.066$. En tanto que los coeficientes de regresión estandarizados estuvieron en el rango .42 a .81 (Tabla 4).

c) Consistencia interna

El coeficiente alfa de Cronbach resultó igual a .871 , lo cual indica un valor aceptable de consistencia interna (Tabla 4).

d) Relación entre compra impulsiva, arrepentimiento y edad

De los 315 participantes que fueron incluidos en los análisis de la Escala de Compra Impulsiva, cuatro no completaron la Escala de Arrepentimiento, por lo cual los siguientes análisis se basan en 311 participantes. Se condujo un análisis para evaluar la relación entre compra impulsiva y arrepentimiento, controlando luego por la edad de los participantes. Los resultados evidenciaron una relación positiva y estadísticamente significativa entre compra impulsiva y arrepentimiento $(r=.158, p=.005)$, aún luego de controlar el efecto de la edad $\left(r_{\mathrm{p}}=.138, p=.015\right)$.

\section{Discusión y Conclusiones}

En este artículo se han expuesto estudios con dos muestras de participantes cuyo propósito fue evaluar las propiedades psicométricas de la Escala de Compra Impulsiva propuesta por Rook y Fisher (1995) en el contexto local. Específicamente, se evaluó la estructura factorial de manera exploratoria y confirmatoria y la consistencia interna en muestras de estudiantes universitarios y adultos de 18 a 65 años de la ciudad de Córdoba (Argentina). Los resultados indicaron que la escala cuenta con buenas propiedades psicométricas y es posible emplearla en el contexto local. Asimismo, se evaluó la relación de la puntuación total en compra impulsiva con una medida general de arrepentimiento, observándose una relación positiva pero baja. A continuación, se discuten los resultados encontrados en el marco de los antecedentes de la escala.

Al analizar la estructura factorial de manera exploratoria en la muestra de estudiantes universitarios (muestra $A$ ), al igual que el estudio de Rook y Fisher (1995), se observó la existencia de una sola dimensión, la cual explica el $37,17 \%$ de la varianza total. Además, todos los ítems presentaron cargas factoriales mayores de .40. En cuanto al análisis confirmatorio, los índices de ajuste fueron semejantes a los observados por los autores de la escala original: $\mathrm{AGFI}=.916, \mathrm{NFI}=.935, \mathrm{CFI}=.968$ para este estudio, y $\mathrm{AGFI}=.92 ; \mathrm{NFI}=.94 ; \mathrm{CFI}=.97$ para el estudio original. Las cargas factoriales estuvieron en el rango de .42 a .78, resultando todas significativas a un nivel $p<.001$.

Tabla 4

Muestra B: población general - Coeficientes de regresión estandarizados de los items de la Escala de Compra Impulsiva $y$ alfa de Cronbach si el item es eliminado

\begin{tabular}{lcc}
\hline & AFC & $\begin{array}{c}\text { alfa de Cronbach si } \\
\text { el ítem es eliminado }\end{array}$ \\
\hline 1. A menudo compro cosas de manera espontánea & .54 & .86 \\
2. "Ya mismo" describe la forma en que compro las cosas & .70 & .85 \\
3. A menudo compro cosas sin pensar & .79 & .84 \\
4. Si veo algo que quiero, lo compro & .58 & .86 \\
5. "Compro primero y pienso después" me describe bien & .76 & .85 \\
6. Algunas veces soy un poco insensato (irracional, ilógico) comprando & .81 & .85 \\
7. Compro cosas según cómo me siento en el momento & .60 & .86 \\
8.r Planifico cuidadosamente la mayoría de las compras & .42 & .87 \\
9. Algunas veces soy un poco imprudente con lo que compro & .68 & .86 \\
\hline
\end{tabular}

Nota. AFC = análisis factorial confirmatorio inicial, coeficientes de regresión estandarizados 
En el estudio original de la escala con estudiantes universitarios, los pesos factoriales estuvieron en el rango de .60 a .81, también todas significativas a un nivel $p<.001$, aunque no es posible realizar una comparación directa con lo observado en esta investigación debido a que no se informa en el artículo de la escala original el método de estimación. No obstante, se destaca la menor carga factorial observada en este estudio en para el ítem 8 ("Planifico cuidadosamente la mayoría de las compras"), el cual es el único con puntuación inversa. Por último, el análisis de confiabilidad a través del coeficiente alfa de Cronbach muestra también semejanzas con el estudio original, con buenos valores en ambos casos, .84 en este estudio y .88 para la versión original.

En el estudio con muestra de población general (muestra B), el análisis confirmatorio arrojó valores de ajuste similares (AGFI = .925; $\mathrm{NFI}=.953 ; \mathrm{CFI}=.972$ ); también se observaron semejantes coeficientes de consistencia interna (alfa de Cronbach $=.87$ ). Adicionalmente, en esta muestra se examinó la relación entre compra impulsiva y arrepentimiento, relación que resultó estadísticamente significativa y mostró ser positiva pero baja $(r=.158)$, manteniéndose aún luego de controlar el efecto de la edad $\left(r_{\mathrm{p}}=.138, p=.015\right)$, en concordancia con lo planteado por Rook (1987) y Spears (2006).

$\mathrm{Si}$ bien los resultados de este estudio evidencian que la Escala de Compra Impulsiva es un instrumento válido y confiable para ser utilizado en la población local, vale señalar algunas limitaciones que originan futuras líneas de acción. En cuanto a aspectos metodológicos, vale remarcar que las muestras fueron no probabilísticas, lo cual limita la posibilidad de generalizar estos resultados muestrales a las poblaciones de referencia. Por ello, futuros trabajos podrían procurar contar con muestras aleatorias.

Un aspecto que tiene implicancias teóricas y metodológicas es la conceptualización de la compra impulsiva. En este trabajo se estudiaron las propiedades psicométricas de una escala clásica para medir la compra impulsiva. No obstante, la definición del constructo está en debate, lo cual se traduce en una multiplicidad de instrumentos que abordan distintos componentes de la compra impulsiva (e. g., Sarabia Sánchez y Schmidt, 2004; Verplanken \& Herabadi, 2001), por lo cual sería recomendable progresar en síntesis integradoras que permitan dar cuenta de las distintas facetas del constructo $y$, a su vez, poder establecer la relación con otros construc- tos tales como la auto-regulación (Verplanken \& Sato, 2011). Asimismo, sería relevante avanzar en la obtención de evidencias de validez ecológica, esto es, indagar si lo que se observa en situaciones controladas son representativas de lo que ocurre en la vida cotidiana (Brewer, 2000).

En resumen, este trabajo ha aportado evidencia de validez de constructo y de consistencia interna de la Escala de Compra Impulsiva de Rook y Fisher (1995) en estudiantes universitarios y adultos de la ciudad de Córdoba, con buenos resultados, lo cual habilita su uso en el contexto local. Se sugiere avanzar en algunas de las líneas antes propuestas para así posteriormente poder generar estrategias de intervención tendientes a promover decisiones más responsables.

\section{Referencias}

Bell, D. E. (1982). Regret in decision making under uncertainty. Operation Research, 30, 5, 961-981.

Brewer, M. B. (2000). Research design and issues of validity. En H. T. Reis, \& C. M. Judd (Eds.), Handbook of research methods in social and personality psychology (pp. 3-16). New York: Cambridge University Press.

Cohen, R. J., y Swerdlik, M. E. (2006). Pruebas y Evaluación Psicológicas. España: Mc Graw Hill.

Comisión de Enlace Institucional, AAM-SAIMO-CEIM (2006). Nivel Socio Económico 2006. Recuperado de http://www.saimo.org.ar/socios/Socios/NSE200623nov2006-Informe_final.pdf

George, D., \& Mallery, M. P. (2001). SPSS for Windows step by step: A simple guide and reference. Boston, MA: Allyn \& Bacon.

Inman, J. J., \& Mcalister, L. (1994). Do coupon expiration dates affect consumer behavior? Journal of Marketing Research, 31, 423-428.

Inman, J. J., \& Zeelenberg, M. (2002). Regret in repeat purchase versus switching decision: The attenuating role of decision justifiability. Journal of Consumer Research, 29, 116-128.

Jones, M. A., Reynolds, K. E., Weun, S., \& Beatty, S. E. (2003). The product-specific nature of impulsive buying tendency. Journal of Business Research, 56(7), 505-511.

Loomes, G., \& Sugden, R. (1982). Regret theory: An alternative theory of rational choice under uncertainty. Economic Journal, 92, 805-824.

Miller, E. (2003). The measurement of impulsivity (Unpublished Doctoral dissertation). University of Warwick, United Kingdom. Recuperado de http://webcat. warwick.ac.uk/record=b1667202 S9.

Pirón, F. (1991). Defining impulse purchasing. Advances in Consumer Reasercha, 18, 509-514.

Reyna, C. E. (2013). Toma de decisiones económicas y procesos emocionales: Análisis instrumental y experimentos en situaciones de consumo individual e interpersonal. (Informe de proyecto). Secretaría de Ciencia y Tecnología, Universidad Nacional de Córdoba 
Rook, D. W. (1987). The buying impulse. Journal of Consumer Research, 14(2), 189-199.

Rook, D. W., \& Fisher, R. J. (1995). Normative influences on impulsive buying. Journal of Consumer Research, 22, 305-313.

Sarabia Sánchez, F. J., y Schmidt, T. H. (2004). Un análisis del comportamiento de impulsividad del consumidor y propuesta de una escala para medir el comportamiento impulsivo. Esic Market, 119, 169-194.

Schwartz, B., Ward, A., Monterosso, J., Lyubomirsky, S., White, K., \& Lehman, D. R. (2002). Maximizing versus satisficing: Happiness is a matter of choice. Journal of Personality and Social Psychology, 83(5), 1178-1197.

Simonson, I (1992). The influence of Anticipating Regret and Responsibility on Purchase Decisions. Journal of Consumer Research, 19, 1, 105-118.
Spears, N. (2006). Just moseying around and happening upon it versus master plan: Minimizing regret in impulsive versus planned sales promotion purchases. Psychology \& Marketing, 23(1), 57-73.

Tabachnick, B., \& Fidell, L. (2001). Using multivariate statistics (4th ed.). New York: Harper \& Row.

Verplanken, B., \& Herabadi, A. (2001). Individual differences in impulsive buying tendency: Feeling and no thinking. European Journal of Personality, 15, S71-S83.

Verplanken, B., \& Sato, A. (2011). The psychology of impulsive buying: An integrative self-regulation approach. Journal of Consumer Policy, 34(2), 197-210.

Youn, S., \& Faber R. J. (2000). Impulsive buying: Its relation to personality traits and cues. Advances in Consumer Research, 27, 179-185.

Para citar este artículo:

Revilla, R. G., Acosta, C. y Reyna, C. (2013). Estudios de validez y confiabilidad de la escala de compra impulsiva en jóvenes y adultos de la ciudad de Córdoba, Argentina. Ciencias Psicológicas VII (2): $133-142$. 